\title{
ARCONS: a Highly Multiplexed Superconducting UV-to-Near-IR Camera
}

\author{
Kieran O'Brien ${ }^{1}$, Ben Mazin ${ }^{1}$, Sean McHugh ${ }^{1}$, \\ Seth Meeker ${ }^{1}$ and Bruce Bumble ${ }^{2}$ \\ ${ }^{1}$ Department of Physics, University of California, Santa Barbara, CA 93106, USA \\ email: kobrien@physics.ucsb.edu \\ ${ }^{2}$ Jet Propulsion Laboratory, Pasadena, California 91107, USA
}

\begin{abstract}
ARCONS, the Array Camera for Optical to Near-infrared Spectrophotometry, was recently commissioned at the coudé focus of the 200-inch Hale Telescope at the Palomar Observatory. At the heart of this unique instrument is a 1024-pixel Microwave Kinetic Inductance Detector (MKID), exploiting the Kinetic Inductance effect to measure the energy of the incoming photon to better than several percent. The ground-breaking instrument is lens-coupled with a pixel scale of $0^{\prime \prime} .23 /$ pixel, each pixel recording the arrival time $(<2 \mu \mathrm{sec})$ and energy of a photon $(\sim 10 \%)$ in the optical to near-IR (0.4-1.1 microns) range. The scientific objectives of the instrument include the rapid follow-up and classification of transient phenomena.
\end{abstract}

Keywords. instrumentation: detectors, instrumentation: spectrographs, pulsars: individual(Crab)

\section{Background}

(a) The Kinetic Inductance Detector. The working principle of the Kinetic Inductance Detector was described in detail in Day et al. (2003), and is summarized here. Photons with energy $h \nu$ are absorbed in a superconducting film, producing a number of excitations, called "quasiparticles" (Fig. 1a). To measure those quasiparticles sensitively, the film is placed in a high-frequency planar resonant circuit (Fig. 1b). Figs. 1c, d show the effect on the amplitude and phase respectively of a microwave excitation signal sent through the resonator. The change in the surface impedance of the film following a photon absorption event pushes the resonance to lower frequency and changes its amplitude. If the detector (resonator) is excited with a constant on-resonance microwave signal, we can measure the degree of phase and/or amplitude shift caused by a single incident optical photon.

(b) Energy resolution. Since the energy of the incoming photon is many times that necessary to generate a quasiparticle, several thousand quasiparticles are generated by each photon (in contrast to a semiconductor, where the incident photon has an energy only slightly above the band-gap). The degree of phase-shift is related to the number of quasiparticles, and hence to the energy of the incoming photon. The maximum theoretical resolution, $R(=E / \delta E)$ is given by,

$$
R=\frac{1}{2.355} \sqrt{\frac{\eta h \nu}{F \Delta}},
$$

where $\eta$ is an efficiency factor, $\mathrm{F}$ the Fano factor and $\Delta$ the superconducting energy gap.

(c) Multiplexing scheme. By engineering each resonator to have a slightly different resonant frequency, a large number (a few thousand) of resonators (pixels) can be probed 

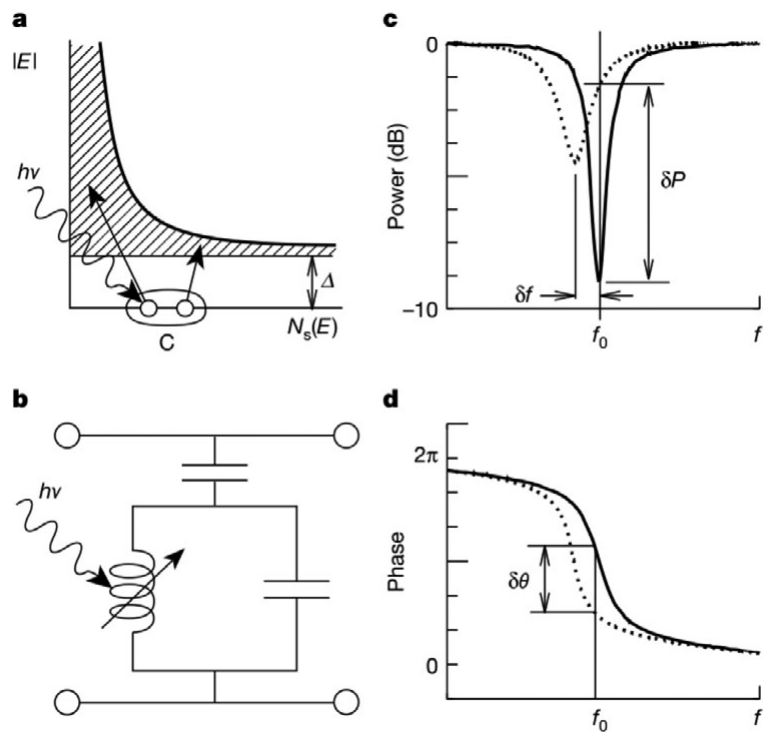

Figure 1. An illustration of the detection principle, from Day et al. 2003.
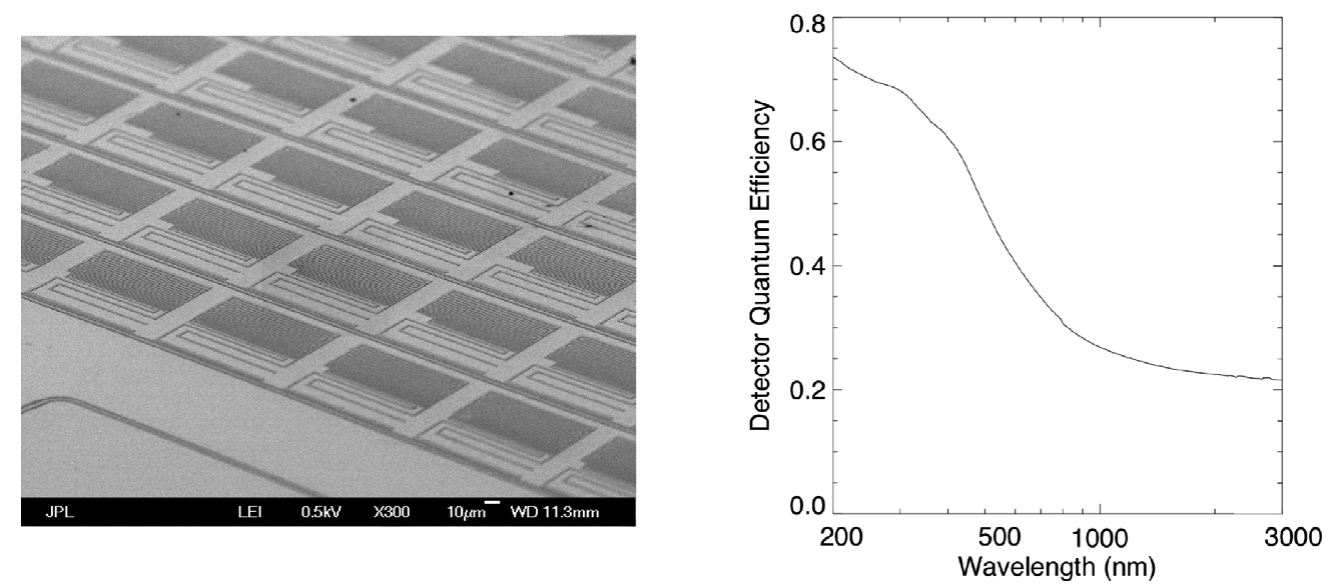

Figure 2. Left: Image of a section of the science array. The individual pixels can been seen to have a slightly different length meandered section in order to tune the resonant frequency, enabling the highly multiplexed read-out. Right: the measured quantum efficiency of the TiN-lumped element detector.

simultaneously by a comb of frequencies sent down a single coaxial line. In the current scheme we use two coaxial cables with 512 resonators on each line in the range $4-5 \mathrm{GHz}$.

\section{The Instrument}

ARCONS (Mazin et al. 2010) uses a cryogen-free ADR to cool an array of TitaniumNitride (TiN)-lumped element MKIDs to a temperature of $85 \mathrm{mK}$ (which is well below the superconductor $T_{c}$ of $\sim 800 \mathrm{mK}$ ). The instrument has a hold time of $\sim 12 \mathrm{hrs}$ before it needs to be regenerated; the latter step takes $\sim 2 \mathrm{hrs}$. The $32 \times 32$ array (shown in Fig. 2) is on a $100-\mu \mathrm{m}$ pitch, which is behind a $200-\mu \mathrm{m}$ focal-length micro-lens array, increasing the fill factor to $64 \%$ and increasing the uniformity by concentrating the light on a small region of the inductor. While the MKIDs are sensitive from the UV to mid-IR 

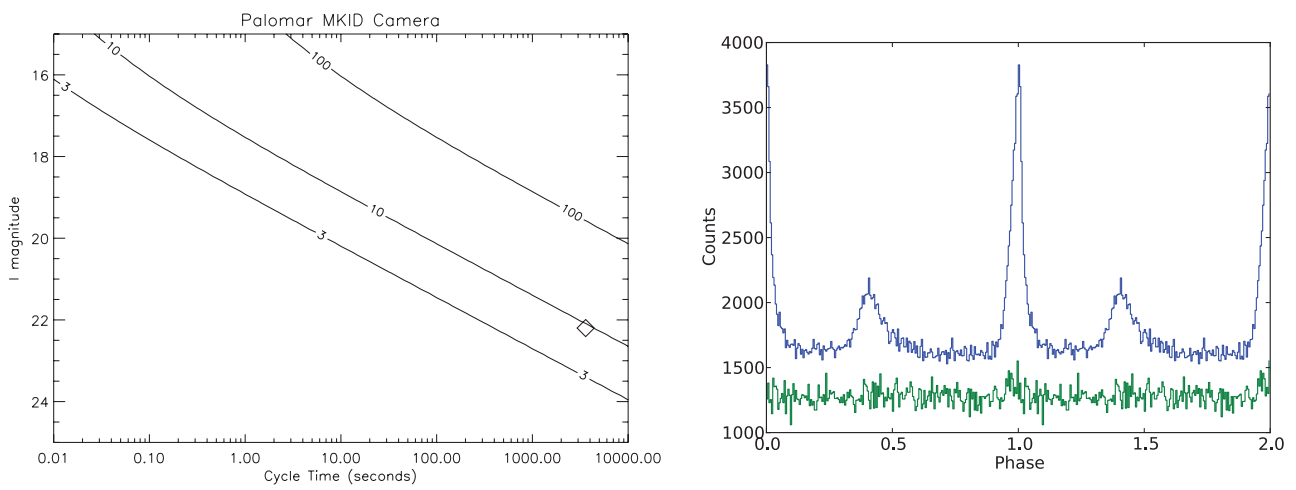

Figure 3. Left: predicted sensitivity of ARCONS at Palomar, with contours for signal-to-noise ratios of 3, 10 and 100. The diamond shows a measurement from the 2011 commissioning run. Right: the phase-folded Crab Pulsar light curve (repeated for 2 cycles of the $33 \mathrm{msec}$ spin period for clarity) from a 20 -sec twilight observation. The sky variability is also shown (offset).

(see Fig. 2), the choice of glass cuts them off below $400 \mathrm{~nm}$ and a filter (ASAHI "supercold") sets the red limit at $1100 \mathrm{~nm}$, limiting the total count-rate (dominated by the sky). The pixels are $0^{\prime \prime} .23$ on the sky, giving a field of view of $\sim 7^{\prime \prime} .5 \times 7^{\prime \prime} .5$. Each pixel has a $\mathrm{R} \sim 12$ at $400 \mathrm{~nm}$, but was strongly affected by "substrate events" where the photon is absorbed in the Si substrate, leading to a breakdown in the relationship between photon energy and phase shift.

The pixels are read out using a custom-built software-defined radio (SDR) system (McHugh et al. 2011), in which a frequency comb is created in software, and up-converted to the required frequency range. That signal is sent along the coax and passes through the MKIDs. The transmitted signal is amplified and then down-converted and digitized via onboard A/D converters. It is then "channellized", and the pulse heights (a direct measure of the photon energy) and pulse start-times (photon arrival times) are measured in a powerful FPGA.

\section{First Light}

ARCONS was successfully commissioned during four nights in 2011 July/August. The throughput was as expected. We observed a broad range of "science demonstration" targets, including interacting binaries (AM Cvns, LMXBs, short-period eclipsing sources), QSOs (for low-resolution redshift measurements), supernovæ (Type Ia and Type II) and the Crab pulsar.

\section{Science Goals}

(a) Rapid characterization of transients. ARCONS will allow us to determine the spectrum of a transient source as it evolves. The small field, readout-noise-free IFU will allow point-and-shoot deep spectroscopic observations. That will enable better marshalling of follow-ups on large-aperture telescopes.

(b) Time-resolved Opt/IR observations of pulsars. Detailed time-resolved spectra, especially if combined with simultaneous radio (GBT) and gamma-ray (Fermi) observations, should reveal details about pulsar emission and the structure of the magnetosphere that will allow us to differentiate between models of optical emission. 
(c) Characterization of short-period variables. There is a growing number of shortperiod variables being discovered by sky surveys, and that number will increase with future large surveys, most notably LSST. These discoveries require detailed follow-up observations in order to characterize the components of the variables. ARCONS is ideally suited for such observations, offering zero dead-time, low-resolution spectroscopy.

(d) Redshift determination via spectro-imaging. We will use the intrinsic energy resolution of the MKIDs to determine the redshift of galaxies out to $z \sim 4$ with a high degree of accuracy. A spectral resolution of 20 in the UV with ARCONS translates into 14 wavelength resolution elements in the wavelength range of the camera. The advantage of ARCONS over multi-filter photometry, such as that employed by COMBO-17, is that all wavelengths are observed simultaneously (thus reducing the exposure time considerably) and through the same conditions (seeing, sky transparency), making the analysis less complicated.

\section{The Future}

With the information and experience we have gained from the recent commissioning run, we have identified a number of upgrades which, when combined, will improve the instrument significantly and promote it from a demonstration instrument to a frontline scientific one. The improvements include increasing the pixel-scale to match better the median seeing at Palomar and to give a wider field of view, increasing the number of pixels and bandwidth of the read-out electronics for reading out a 2048 pixel array, and improving our calibration scheme. In the longer term, we are developing an antireflection scheme to improve the QE of the detectors. MKIDs promise to revolutionize many areas of astronomy, not limited to the time domain, as they are capable of producing large area, read-noise-free integral-field spectroscopy without the need for complicated optical systems. In the time domain, they will offer deep, low-resolution integral-field spectroscopy with the advantage of time-tagging the arrival time of each photon.

\section{Acknowledgements}

We would like to thank the management and staff of Palomar Observatory for their hard work and support during the commissioning of ARCONS. Material in this paper is based upon work supported by the National Aeronautics and Space Administration under Grant NNX09AD54G, issued through the Science Mission Directorate, Jet Propulsion Lab's Research \& Technology Development Program, and a grant from the W.M. Keck Institute for Space Studies. Part of the research was carried out at the Jet Propulsion Laboratory, California Institute of Technology, under a contract with the National Aeronautics and Space Administration.

\section{References}

Day, P., Leduc, H., Mazin, B., Vayonakis, A., \& Zmuidzinas, J. 2003, Nature, 425, 817

Mazin, B., et al. 2010, in: Proc. SPIE, Vol. 7735, 773518

McHugh, S. 2011, Review of Scientific Instruments, submitted. 